\title{
High harmonic generation spectroscopy via orbital angular momentum
}

Cite as: J. Chem. Phys. 151, 084308 (2019); https://doi.org/10.1063/1.5115152

Submitted: 14 June 2019 . Accepted: 06 August 2019 . Published Online: 29 August 2019

Jan Troß (D), and Carlos A Trallero-Herrero (D)

\section{COLLECTIONS}

Paper published as part of the special topic on Ultrafast Spectroscopy and Diffraction from XUV to X-ray Note: This paper is part of the JCP special collection on Ultrafast Spectroscopy and Diffraction from XUV to X-ray.
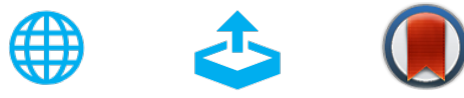

\section{ARTICLES YOU MAY BE INTERESTED IN}

Production of ultracold ${ }^{85} \mathrm{Rb}^{133} \mathrm{Cs}$ molecules in the lowest ground state via the $\mathrm{B}^{1} \Pi_{1}$ shortrange state

The Journal of Chemical Physics 151, 084303 (2019); https://doi.org/10.1063/1.5108637

\section{Scattering off molecules far from equilibrium}

The Journal of Chemical Physics 151, 084301 (2019); https://doi.org/10.1063/1.5111979

Tracing charge transfer in argon dimers by XUV-pump IR-probe experiments at FLASH The Journal of Chemical Physics 151, 084314 (2019); https://doi.org/10.1063/1.5116234

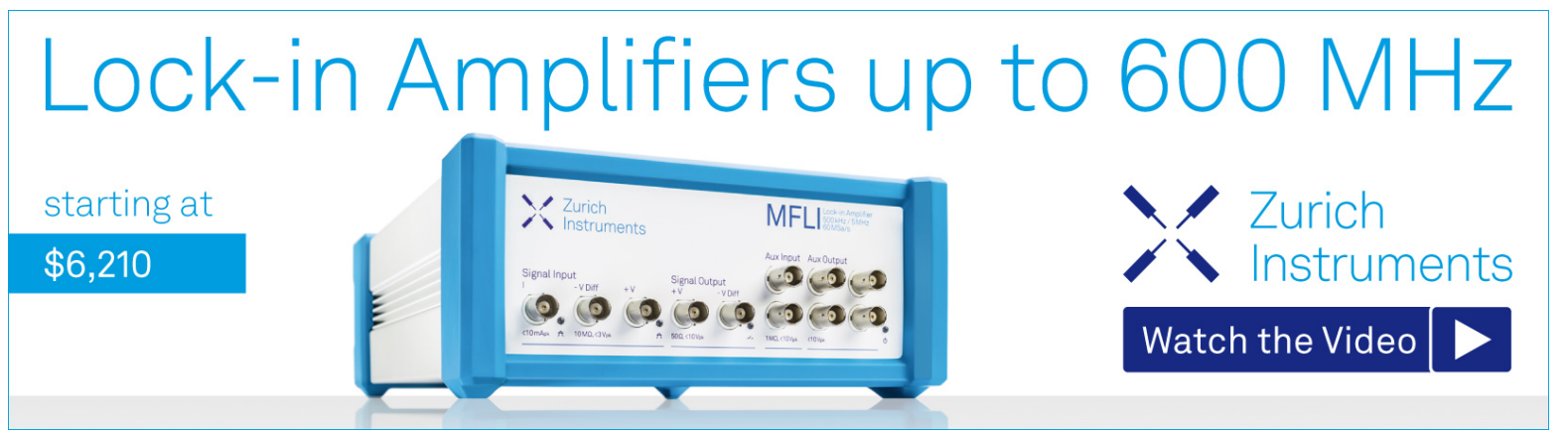




\title{
High harmonic generation spectroscopy via orbital angular momentum
}

\author{
Cite as: J. Chem. Phys. 151, 084308 (2019); doi: 10.1063/1.5115152 \\ Submitted: 14 June 2019 - Accepted: 6 August 2019 • \\ Published Online: 29 August 2019
}

\author{
Jan Tro $\beta^{1}$ (D) and Carlos A Trallero-Herrero
}

\begin{abstract}
AFFILIATIONS
'James R. Macdonald Laboratory, Department of Physics, Kansas State University, Manhattan, Kansas 66506, USA

${ }^{2}$ Department of Physics, University of Connecticut, Storrs, Connecticut 06269-3046, USA
\end{abstract}

Note: This paper is part of the JCP special collection on Ultrafast Spectroscopy and Diffraction from XUV to X-ray.

a) Electronic mail: carlos.trallero@uconn.edu

\begin{abstract}
We present an experimental technique using orbital angular momentum (OAM) in a fundamental laser field to drive high harmonic generation (HHG). The mixing of beams with different OAM allows us to generate two laser foci tightly spaced which generate harmonics that interfere in the far field. Thus, this technique is an OAM based in situ HHG interferometric spectroscopic method. With this tool, we measure the phase and amplitude of the angle dependent multiorbital HHG emission in molecular nitrogen.
\end{abstract}

Published under license by AIP Publishing. https://doi.org/10.1063/1.5115152

\section{INTRODUCTION}

Higher-order harmonic generation $(\mathrm{HHG})^{1-5}$ has been shown to be a powerful spectroscopic technique ${ }^{6-14}$ and has been used for 20 years to push atomic and molecular physics to the attosecond regime, unraveling molecular dynamical structures ${ }^{6}$ and charge migration $^{15}$ at these time scales.

Harnessing the coherence of light and specifically of the emitted higher harmonics, this letter shows another approach for measuring complex signals $S(\omega, t)=|S(\omega, t)| e^{i \phi(\omega, t)}$ in the extreme ultraviolet (XUV) from HHG, which then can be used to study structural details of diatomic nitrogen. More specifically, XUV light emitted through HHG holds complex-valued information about the photorecombination dipole matrix element $d(\omega, \theta)=|d(\omega, \theta)| e^{i \phi(\omega, \theta)}$, where the recombination process is understood as the time reversal of photoionization. ${ }^{16}$ The time reversal relationship between photoionization and photorecombination is better understood through the principle of detailed balanced, ${ }^{17}$ a principle that has been already applied to the description of the generation of harmonics. ${ }^{18,19}$ The relevance of the photoionization dipole lies, of course, in the fact that it has been widely used by chemists and physicists as a tool to investigate atomic and molecular structures. However, in the weak field regime of photoionization, phase information about the transitions cannot be easily retrieved.
In contrast, HHG offers direct access to the amplitude and phase of the photoionization dipole. Calculations of the photorecombination dipole aided by experiments have been presented in nitrogen ${ }^{20}$ and show energy and angle dependent variations. A shape resonance at $30 \mathrm{eV}$ shows dramatic enhancement of the cross section and a variation of the phase as a function of energy. Measurements of the angle and energy dependent cross section have been measured through other techniques such as the reconstruction of attosecond beating by interference of two-photon transitions (RABBITT). ${ }^{21,22}$ In $\mathrm{N}_{2}$, phase information has been investigated ${ }^{23}$ in RABBITT and through interferometric studies ${ }^{24,25}$ with separate beam paths, as well.

However, even with table-top techniques such as RABBITT, extracting these features is experimentally challenging and requires a stable interferometric setup. This is where our proposed experimental techniques come into play. Using a programmable spatial light modulator (SLM), we can build a stable, reliable commonpath interferometer that is insensitive to instabilities in experimental setups.

We use the well documented features of diatomic nitrogen to benchmark our technique of using beams with orbital angular momentum (OAM), while studying the angle dependence of individual harmonics with molecular alignment. In nitrogen, similar measurements have already been performed and showed reliable 
features with different details. In a 2015 publication, Camper et al. ${ }^{2}$ used a binary phase mask to study the change in phase of harmonics as the molecules rotate in time and shows for harmonics 9-17 an oscillatory behavior of the phase, while a measurement by Lock et al. ${ }^{24}$ showed no change in phase for harmonic 19 . The key difference in the experiments is the way two intense laser fields are produced. We will show results that also extend the measurements to the molecular frame. Furthermore, publications ${ }^{26,27}$ have also shown the presence of multiple orbitals to the harmonic spectra and their direct comparison to the double differential cross section. ${ }^{19,28}$ When using molecules as targets, the electronic configuration can be described with molecular orbitals, ${ }^{29,30}$ where the electron in the highest occupied molecular orbital (HOMO, $3 \sigma_{g}$ for $\mathrm{N}_{2}$ ) has the smallest binding potential with the molecule and the next lower lying orbital is defined as the HOMO- $1\left(1 \pi_{u}\right)$. In nitrogen, these two orbitals have ionization potentials of $15.6 \mathrm{eV}$ for the $\mathrm{HOMO}$ and $16.9 \mathrm{eV}$ for the HOMO-1. These binding potentials are very close to each other, and ionization rates can become similar which needs to be taken into account when using HHG as a spectroscopic tool. $^{28}$

\section{EXPERIMENTAL SETUP}

We use a pump-probe scheme, where the pump pulse is inducing molecular alignment and the probe is driving high harmonic generation, which is used as our spectroscopic tool. The relative delay between pump and probe pulses is controlled by changing the optical beam path of the pump pulses by moving a mirror on a motorized linear stage. The experimental setup is shown in Fig. 1. In this setup, the probe arm is reflected off a beam splitter and is shaped by a spatial light modulator (SLM) to form a two source focus spot. The probe is focused by an $\mathrm{f}=75 \mathrm{~cm}$ lens into the gas jet, and harmonics are generated by both focus spots. As the harmonics travel into the far field, harmonics from the two sources interfere and form a fringe pattern on the detector, which allows us to detect the relative phase between the harmonic sources and their intensity.
The pump arm is transmitted through the first beam splitter and contains initially $80 \%$ of the total pulse energy. After shrinking the beam by a factor of two with a telescope, the beam is split again by a set of two beam splitters in another interferometer inside the pump arm. By controlling the relative delay between the two pump pulses, we can tune the interaction of the pumps upon the molecular target and enhance the molecular alignment ${ }^{31-33}$ or potentially orient molecules in space. ${ }^{34,35}$ The pump beams are focused with a separate lens of $\mathrm{f}=50 \mathrm{~cm}$ into the center of the gas jet, as seen in Fig. 1. The pump and probe beams are recombined with a beam splitter. The focus of the pump beam is placed at the center of the jet and is overlapped with one of the two probe beams.

The spot size of the pumps is measured to be $68 \mu \mathrm{m}$ by $83 \mu \mathrm{m}$ and hold a pulse energy of $200 \mu \mathrm{J}$ with a pulse duration of $100 \mathrm{fs}$, measured by a cross correlation between the measured probe of $30 \mathrm{fs}$ and the pump beams, which results in an experimentally determined pump intensity of $21 \mathrm{TW} / \mathrm{cm}^{2}$ and a probe intensity of $110 \mathrm{TW} / \mathrm{cm}^{2}$. The overlap between the pump and probe is optimized on the live harmonic signal, and we check that only one of the two sources is interacting with the pump beams (by using different sets of phase masks as highlighted in Section III). Switching between masks is also used to find an optimum overlap between the gas jet and the two probe beams. The gas jet ${ }^{36}$ has a nozzle of $300 \mu \mathrm{m}$ after which the gas expands over $3 \mathrm{~mm}$ before able to interact with laser beams. The vertical offset is adjusted with a micrometer on the three dimensional manipulator, holding the jet. In the data acquisition, amplitude and phase of the harmonics are collected as a function of delay between the pump and probe in step sizes of $40.04 \mathrm{fs}$. This step size is a sufficient sampling in time to capture smallest features in the revival structure from nitrogen, with a rotational period of 8.3 ps. In the later experimental data, we can see smaller features with periods of $240-400$ fs that can be resolved with the given step size.

As briefly mentioned before, a SLM is implemented to create a two-foci intensity distribution that in turn produces an interferometric pattern of the XUV pulses in the far field. Instead of using phase patterns that break the spatial symmetry ${ }^{11,25}$ of the

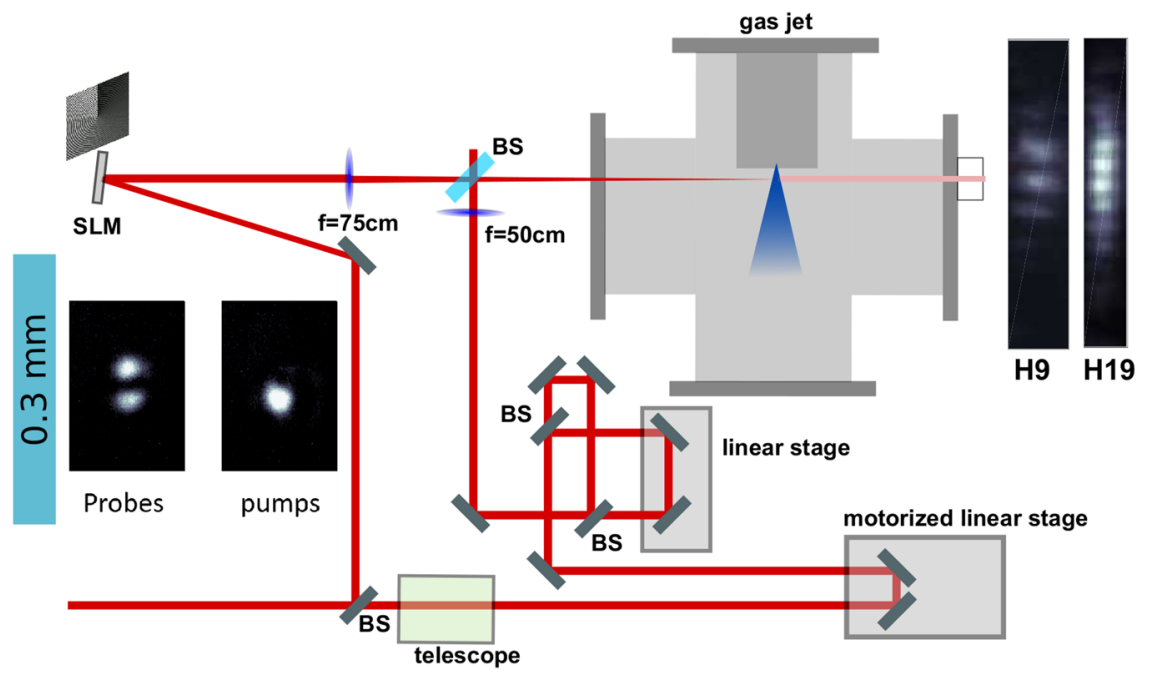

FIG. 1. Experimental setup with an unbalanced Michelson interferometer to separate the input into one probe and two pump arms. The probe and pump beams are focused into a pulsed gas jet, in which high harmonics are generated from the two foci of the probe. A dual focus is achieved by superposing beams with $\mathrm{OAM} I= \pm 1$. XUV light generated from the two foci interferes in the far field creating the pattern shown in the right inset for $\mathrm{H} 9$ and $\mathrm{H} 19$. In the left inset, pump and probe foci are shown in respect to the nozzle diameter of $300 \mu \mathrm{m}$, preparing the pulsed gas sample before it interacts with the laser focus after $3 \mathrm{~mm}$ of travel and expansion. 
incoming beam, orbital angular momentum (OAM) is applied to generate two foci, imprinted by the whole surface of the SLM. The benefit of this approach is that the entire incoming beam will be affected by the phase distribution at the SLM which translates into two beams that are indistinguishable up to millimeters away from the interaction region. As a result, the XUV interferometer is completely self-referencing and insensitive to air currents and optics imperfections since both light paths have the exact same optical path.

\section{DETAILS OF SPATIAL LIGHT SHAPING}

For completeness, we include details of the Fourier optics calculation that demonstrates the generation and control of two foci. Details of such methods can be found elsewhere. ${ }^{37}$ When monochromatic light with a spatial profile of $U(\eta, v)$ propagates from the source plane $(\eta, v)$ to the observation plane $(x, y)$, we can formulate the propagation with an impulse function $h$ that can be simplified to a simple propagator $h=\frac{e^{i k r}}{r}$. The field $U^{\prime}(x, y)$ in the observation plane can be predicted $\stackrel{r}{r}$ using the first Rayleigh-Sommerfeld diffraction solution

$$
U^{\prime}(x, y)=\frac{z}{i \lambda} \iint U(\eta, v) \frac{\exp (i k r)}{r^{2}} d \eta d v,
$$

with $\lambda$ being the wavelength, $k$ being the wave number, and $z$ being the distance between the source and the observation plane. In Eq. (1), $r$ is the distance between a position on the source plane and a position on the observation plane, $r=\sqrt{z^{2}+(x-\eta)^{2}+(y-v)^{2}}$. Different approximations for the square root term result in the Fresnel (and Fraunhofer) diffraction formulas, giving solutions to the diffraction through an aperture with the characteristic size $a .^{37} \mathrm{We}$ choose the Fraunhofer approximation based on the Fresnel number $N=\frac{a^{2}}{L \lambda}$, with $L$ being the distance to the observation plane (focus) from the aperture (SLM). Equation (1) can be used to calculate the focal spot of an arbitrary spatially shaped pulse or, in our case, beams that have the purpose of generating two intense spots to generate harmonics.

While the generation of harmonics driven by beams with OAM has been demonstrated, ${ }^{38}$ exploring multifoci experiments with OAM for HHG has not been demonstrated. In a previous publication, we used beams with superposed OAM for micromachining purposes ${ }^{39}$ proving that intense multifoci femtosecond beams with OAM can be generated.

\section{A. Orbital angular momentum}

Using the aspect of OAM to shape the focus of our Gaussian beam into two separate foci is the key to a stable interferometer. Throughout the paper, we use the definition of Laguerre Gaussian (LG) beams, as in Ref. 40. LG beams are an equivalent description of transverse electromagnetic modes, but in cylindrical rather than Cartesian coordinates, and these modes carry the so-called orbital angular momentum. A strict definition of LG modes requires Laguerre polynomials and different normalization constants. However, we use a Gaussian mode that has OAM and shares in this regard similarities with LG beams.
We write a Gaussian beam as

$$
U(\rho, z)=E_{0} \frac{w_{0}}{w(z)^{2}} \exp \left[-\frac{\rho^{2}}{w(z)^{2}}-i\left(k z+k \frac{\rho^{2}}{2 R(z)}-\Phi(z)\right],\right.
$$

with $E_{0}$ being the peak field strength, $w_{0}$ being the initial beam waist, $w(z)$ being the beam waist at position $z$, $\rho$ being the radius, $R(z)$ being the radius of curvature of the wavefront, and $\Phi(z)$ being the Gouy phase. To this Gaussian distribution, the SLM adds a phase term that carries OAM, $\exp (i l \theta)$,

$$
U^{*}(\rho, \theta, z)=U(\rho, z) \exp (i l \theta) .
$$

Experimentally, the SLM generates beams with $l=1$ and beams with $l=-1$, resulting in a phase difference between the two different LG modes of $\Delta \Phi(\theta)=2 \theta$. The phase mask and intensity profile used to simulate our beam propagation is shown in Figs. 2(a) and 2(b), respectively. Generation of two superposed OAM beams with $l= \pm 1$ can be thought of as two identical independent waves with different phases $U_{1}=|U| e^{i \phi_{1}}$ and $U_{2}=|U| e^{i \phi_{2}}$ that when overlapped in space generate a space-dependent interference profile given by

$$
I=2 U^{2}(1+\cos \Delta \phi),
$$

with $\Delta \phi=\phi_{2}-\phi_{1}$. The simulated intensity distribution at the focus of a $75 \mathrm{~cm}$ focal length lens has phase and intensity profiles shown in Figs. 2(c) and 2(d), respectively. Constructive interference occurs at integer multiples of $\Delta \phi=2 l \pi$ or at the maximum two lobes in Fig. 2(d). At values of $\Delta \phi=(2 l+1) \pi$, the two light beams interfere destructively which shows up as a minimum in the same figure. This beam profile is similar to what has been previously reported in Refs. 41-44 where a single Gaussian TEM $_{00}$ mode is manipulated by inserting a phase plate with a phase difference of $\pi$ between the upper and lower halves, resulting in two beams parallel to the optical axis or a beam similar to a quasi-Hermite Gaussian of order $\mathrm{TEM}_{01}$, but only through the alternating sampling of OAM with opposing sign, can we achieve a stable mode. Inserting a phase plate of two zones does not guarantee a stable mode as beam pointing instabilities would result in a changing intensity ratio and interference condition.

The SLM allows yet finer control over the spatial profile of the focused beams. For example, often one desires to have a single beam experiment to serve as a reference. In such cases, the one focus should be identical to a single focus of the multibeam experiment. To achieve this, we generate a superposed beam with OAM $1=0,1$ (or -1). To understand how this approach works, Eqs. (3) and (4) are used. At the focal plane, the phase difference between the beam with $l=0$ and the beam with $l=1$ is

$$
\Delta \phi(\theta)=\phi_{1}-l \theta-\phi_{2},
$$

where the beam with $l=0$ is again a pure Gaussian mode. For $l=1$, there is only one possible value for destructive interference and thus a single mode focus is created. This is clear in Fig. 2 panels (e) and (f), where a beam with OAM $l=0,1$ is simulated under the same conditions as before. Panel (e) shows the phase of the beam at the focus showing a fairly flat spatial profile across the beam, and panel (f) shows the intensity profile with a single mode. However, to improve the generation of an identical copy of the individual 

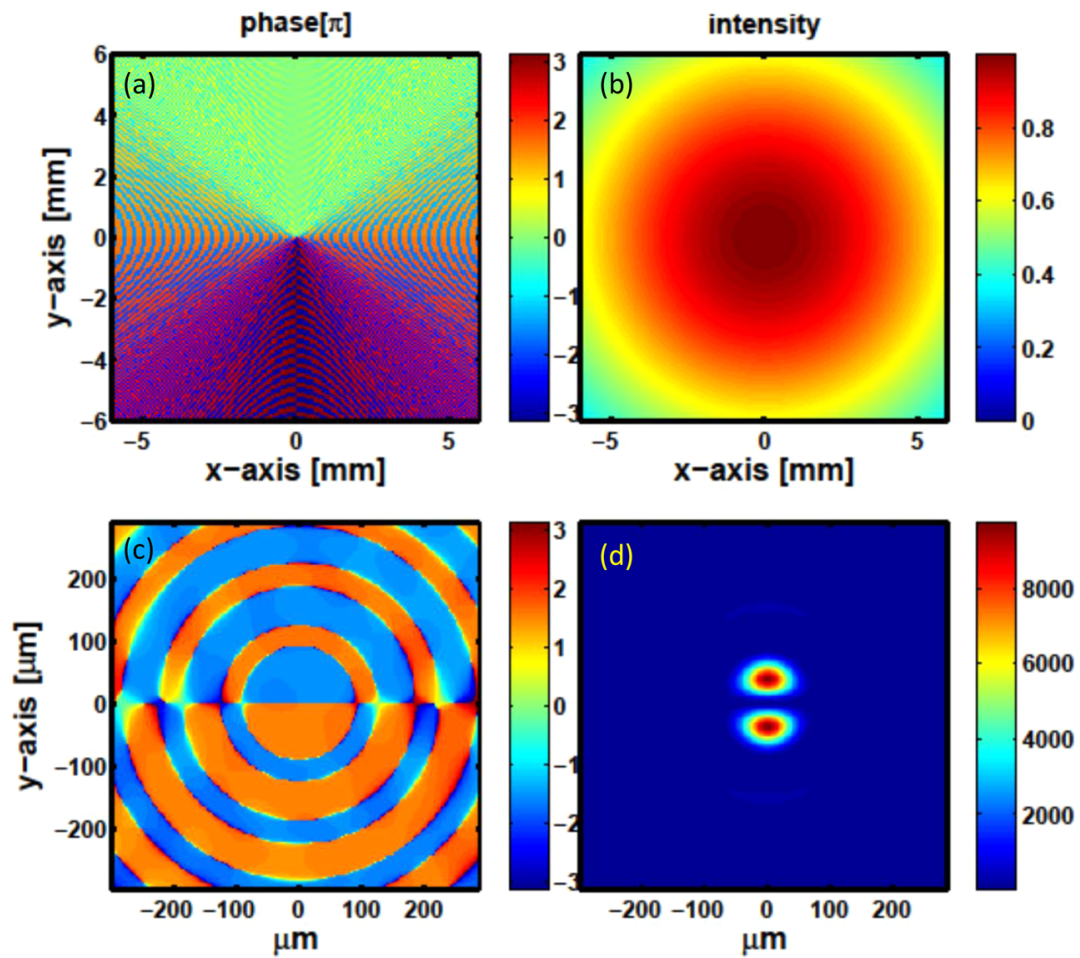

FIG. 2. Panel (a) and (b): Spatial phase and intensity distribution, respectively, in the source plane (SLM) after manipulation of the phase with OAM I = 1, -1 . (c) Spatial phase and (d) spatial intensity distribution at the focus of a $75 \mathrm{~cm}$ lens with a phase mask on the source plane shown in (a) as given by Eq. (3). (e) Phase and (f) intensity spatial distribution at the focus of a $75 \mathrm{~cm}$ lens after manipulation of the phase with $\mathrm{OAM} /=1,0$.
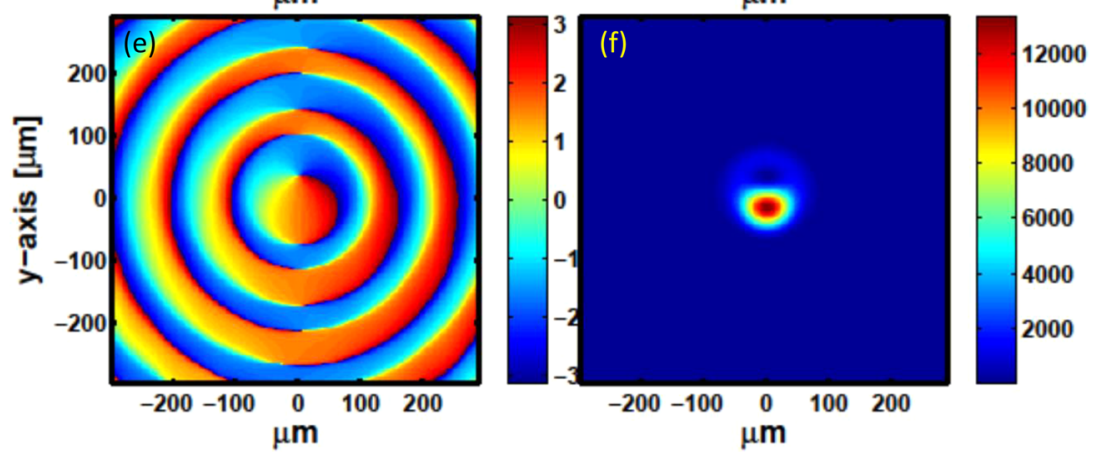

sources, we are faced with the issue that the superposition of beams with OAM $l=1,0$ does not form the same constructive interference as beams with OAM $l= \pm 1$ but forms the constructive interference closer to the optical axis. Imposing a flat wave front tilt on the beam as described in Ref. 37 results in an optimized overlap between single and two source patterns. We found that the tilt applied to the wave front is on the order of 10's of $\mu \mathrm{rad}$. Figure 3 shows a quantitative comparison between a two source $(l= \pm 1)$ and a single source $(l=1,0)$ intensity distribution at the focus of a $75 \mathrm{~cm}$ focal length lens for three different values of tilt. From the figure, we can see that the tilt actually guarantees an overlap between one of the two peaks and a single peak distribution. The left shoulder for $l=1,0$ is almost an order of magnitude lower in intensity than the main peak and thus will have a negligible effect for HHG, for example. Finally, we should mention that unlike previous work done with SLMs for the generation of interferometric HHG spectroscopy ${ }^{15}$ or with glass wedges, ${ }^{25}$ in our method, we use a complete-beam mask that prevents any break in the symmetry of the mode. For example, in previous work where a top-down phase is applied, very small changes between the optical paths on the top part of the beam respect to the bottom will result in measured phase differences in the interference pattern. Applying a phase mask to the entirety of the beam prevents such differences. Still, since both masks make use of interference at the focus to generate two foci, both are limited in the amount of control. To overcome this limitation, we have recently proposed a two-foci mask where the optical phase of the fundamental can also be controlled.

In Fig. 4, we compare the mentioned calculations and experimental observations. Imaging of the modes was done with a CCD camera that was moved with a motorized linear stage. In the figure, beam waist calculations of $\left|U^{\prime}(x, y)\right|^{2}$ are shown as a function of distance around the focus of a $75 \mathrm{~cm}$ lens and measure the spot size in 


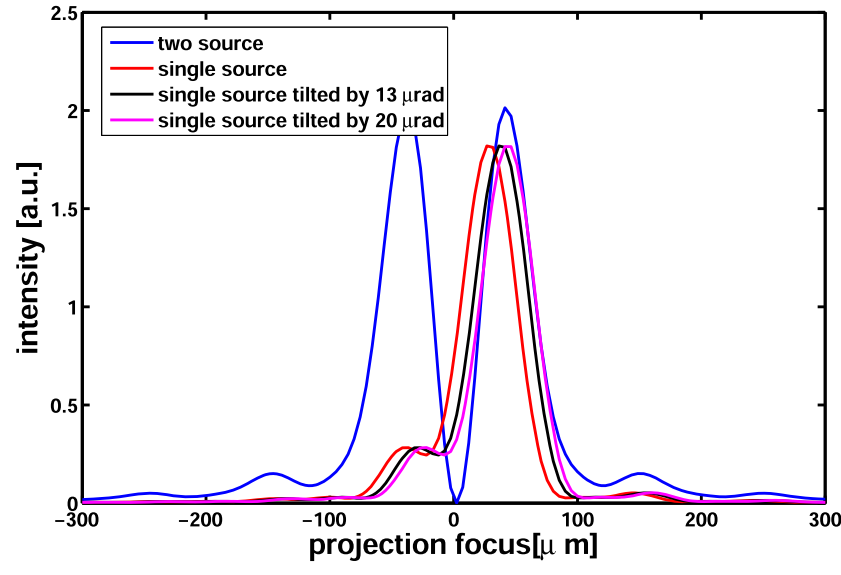

FIG. 3. Comparison between a two source $(I= \pm 1$, blue) and a single source $(I=1,0)$ intensity distribution at the focus of a $75 \mathrm{~cm}$ focal length lens for three different values of tilt (no tilt red, $13 \mu \mathrm{rad}$ black, and $20 \mu \mathrm{rad}$ magenta). The tilt is applied across the entire SLM screen.

the experiment. On the left, we show experimental findings, while on the right, we show the theoretical calculations for the same phase manipulations. In panels (c) and (d), we use $l= \pm 1$ yielding two foci. Panels (a) and (b) and (e) and (f) where generated using $l=1,0$. The only difference between the two sets of panels is an offset applied to the flat phase mask with either $\phi_{2}=0$ or $\phi_{2}=\pi$. This also selects the possible condition for destructive interference, besides the sign convention in $l \theta$. On the top two panels, the $\phi_{2}=0$ point is chosen such that the single focus coincides with the top beam in panels (c) and (d), and in the lower two panels, the single focus coincide with the bottom beam in (c) and (d).

\section{MOLECULAR ALIGNMENT}

As seen in the Quantitative Rescattering (QRS) model, ${ }^{46}$ the harmonic yield of molecules in the molecular frame depends on the angle between the molecular axis and the laser polarization axis. The molecule's axis is given by the molecular frame $z$-axis, and the pump and probe laser polarization is defined by the laboratory frame $Z$-axis. In HHG, the dominant ionization channel is the valence shell or the HOMO of the target atom or molecule. The specific symmetries and dynamics of this particular orbital will then be encoded in the yield of higher harmonics. In nitrogen, the HOMO has the symmetry of a $\sigma_{g}$ orbital, which has an angular density distribution that aligns with the molecular axis and has two nodes perpendicular to the molecular axis. This symmetry would be also visibly recorded on the higher harmonic yield if the molecule are aligned and rotated in space. This angle dependence is due to the molecule's angular dependent ionization and photorecombination rate in the molecular frame. We use an ansatz suggested in Ref. 47 to describe the angle dependent signal of nitrogen as a sum of $\cos ^{2 n} \theta$-terms and $\sin ^{2} \theta \cos ^{2 n} \theta$-terms. In our expansion, terms of order $\sin ^{2} \theta \cos ^{2 n} \theta$ can be expressed as the difference between higher order $\cos ^{2 n} \theta$ terms, which reduces the expansion to $\cos ^{2 n} \theta$-terms. By adding an

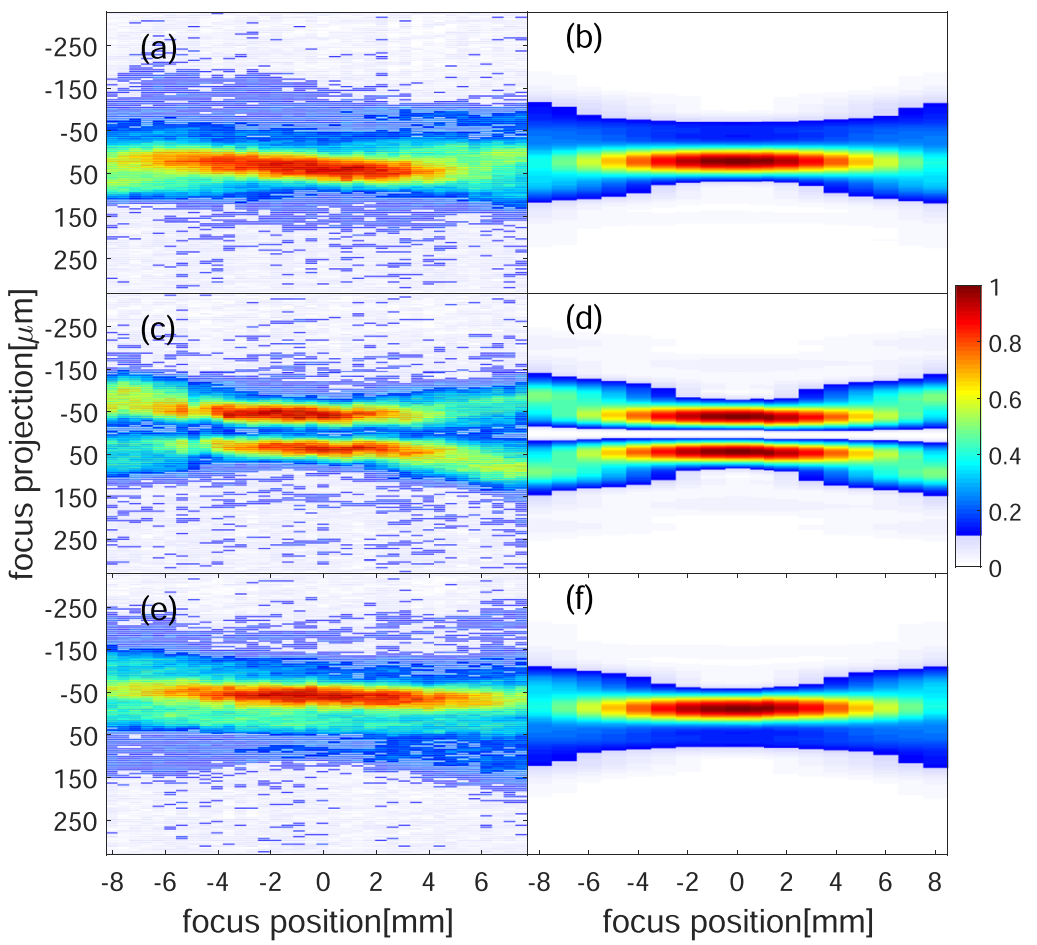

FIG. 4. Panels (a) and (b): Projected focus of a laser beam, manipulated by a phase mask that introduces two beams with $O A M I=1,0$. We observe a single focus with a center of mass similar to the center of mass of one of two foci in panels (c) and (d), where we projected the focus of a laser beam, manipulated by a phase mask that introduces two beams with OAM $I= \pm 1$. In panels $(\mathrm{e})$ and $(\mathrm{f})$, we show the projected focus of the other laser focus. 
appropriate amount of terms of order $n$ to the expansion, we will be able to describe the correct angle dependence. The angle dependent yield $S(\theta, \omega)$ of a harmonic with energy $\hbar \omega$ in the molecular frame is formulated as

$$
S(\theta, \omega)=\sum_{n} C_{n}(\omega) \cos ^{2 n} \theta,
$$

where the signal in the laboratory frame $S(\theta, \omega)$ is the convolution of the molecular frame signal over the molecular axis distribution. The molecular axis has a distribution function that depends on the nonadiabatic alignment of the molecule. The molecular axis distribution $\rho$ is defined by

$$
\rho(\theta, t)=g_{i} \frac{e^{E_{i} / k T}}{Z}\left|\Psi_{i}(\theta, t)\right|^{2},
$$

where $i=\left\{J_{0}, M_{0}\right\}$ is the quantum numbers of the involved states, $g$ is the nuclear spin state weights, $k$ is the Boltzmann constant, $T$ is the rotational temperature, and $Z$ is the partition function. The molecular axis distribution $\rho$ can be interpreted as a probability function of finding the molecule at time $t$ aligned at the angle $\theta$. The distribution can be calculated for the experimentally given laser pulse and gas parameters.

In the laboratory frame, the time dependent signal is defined as an integral over all angles $\theta$,

$$
\begin{aligned}
S(t) & =\int \rho(\theta, t) S(\theta) \sin \theta d \theta, \\
& =\sum_{n} C_{n} \int \rho(\theta, t) \cos ^{2 n} \theta \sin \theta d \theta,
\end{aligned}
$$

where the solution to the integral for a particular order $n$ yields

$$
\int \rho(\theta, t) \cos ^{2 n} \theta \sin \theta d \theta=\left\langle\Psi_{i}\left|\cos ^{2 n} \theta\right| \Psi_{i}\right\rangle(t) .
$$

Each term of the expansion is averaged over the molecular axis distribution. Using this in the time dependent expansion given through Eq. (9), the measured signal $S(\omega, t)$ is then

$$
S(\omega, t)=\sum_{n} C_{n}\left\langle\Psi_{i}\left|\cos ^{2 n} \theta\right| \Psi_{i}\right\rangle(t) .
$$

The coefficients $C_{n}$ are complex and follow the equation $C=A+i B$. Changing the parameters in Eq. (7), in multiple linear regression fits, will result in the residual being minimized. This results in more confidence on the molecular axis distribution. For the smallest residue, the coefficients $C_{n}$ can be inserted in the angle-dependent expansion in Eq. (6) and will define the extracted molecular frame harmonic signal $S(\omega, \theta)$.

\section{EXPERIMENTAL RESULTS}

The experimental yields of harmonics, for alignment with a weak, nonresonant $785 \mathrm{~nm}$ pulse and driven by a delayed $785 \mathrm{~nm}$ probe pulse, are shown in Fig. 5. In the blue, the harmonic yield is given for an experimental condition, where the pump and the probe pulse were spatially overlapped. For the experimental condition yielding the data in the red, no second weak field was present.

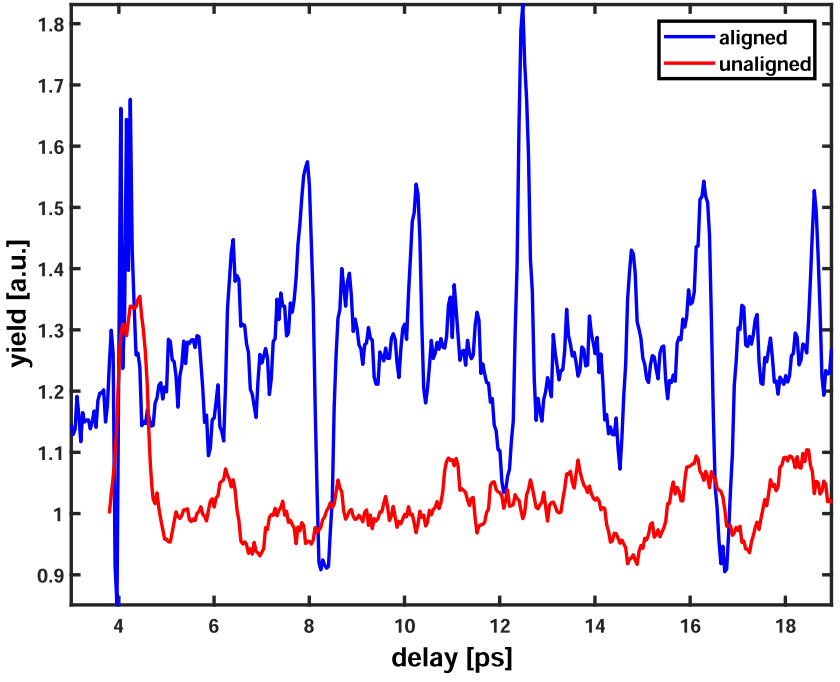

FIG. 5. Harmonic yield as a function of time between the pump and probe, generated by a shaped focus to match the focus of the individual "slits" top and bottom in the experiment.

We observe a periodic time dependence for the blue data set. The harmonic yield strongly depends on the time between the pump and probe. At $8.3 \mathrm{ps}$, the yield is reduced to a value of 0.9 , and at $12.1 \mathrm{ps}$, the yield increases to a value of 2 . The yields are normalized to their isotropic value when no alignment beam is present. We observe quarter revivals at $6.1 \mathrm{ps}, 10.2 \mathrm{ps}$, and $14.2 \mathrm{ps}$. $1 / 8$ revivals are visible at 5 ps, 7 ps, 9 ps, and 11 ps. The unaligned source, in red, where no spatial overlap is visible between the pump and probe in the imaging setup, shows no periodicity in time. We do, however, observe a cross-correlation feature at 4 ps when the pump and probe are incident at the same time. To extract the molecular frame signal, the linear regression is based on the experimental data after the interaction with the pump pulse and the cross-correlation peak does not influence the real physical observations between the unaligned reference source and the aligned second source. In the shown data, the pump beam was spatially aligned to the top spot of the interferometer focus, but the probe beam was spatially shaped to overlap with either the top or bottom spot of the interferometer, leading to a time dependent yield or a time independent yield. After the check of the individual sources and that no rotational alignment is visible in the source with no pump pulse, the pattern is changed to the two-source interference mask and the harmonic yield and phase is collected as a function of time between the pump and probe. The yield from the aligned source is normalized to the total yield of both sources $I_{\text {total }}$,

$$
I_{\text {total }}=I_{1}+I_{2}+2 \sqrt{I_{1} I_{2}} \cos \left(2 \pi \frac{\delta Y}{\lambda z}\right)
$$

where $Y$ is the ordinate of the fringe projection, $I_{1,2}$ is the intensities of the harmonic sources, $\lambda$ is the wavelength of the light, and $z$ is the distance to the observation plane. Integrating over ordinate $Y$, the fringe-angle-integrated yield is equal to $I_{\text {total }}=I_{1}+I_{2} . I_{1}$ and $I_{2}$ are identical sources when no aligning pump beam is present. The 
isotropic yield of the individual harmonic source is then defined to be $I_{1,2, \text { iso }}=I_{\text {total }} / 2$. The delay dependent yield measurement is normalized to this isotropic value, and the intensity of a single source as a function of time can be expressed. To calculate the amplitude of this source, we take the square root of the intensity. In Fig. 6, the time dependent amplitude of harmonic 19, normalized to the isotropic value, is shown. The experimentally collected data show an amplitude of harmonic 19 between 0.5 and 2, when normalized to the isotropic value. At a time of $4.1 \mathrm{ps}$ and $12.3 \mathrm{ps}$, we see a strong antialignment dip in the harmonic amplitude, while we observe a maximum in the recorded yield at 8.2 ps. Besides quarter revivals at $2.1 \mathrm{ps}$ and $6.1 \mathrm{ps}$, smaller revivals in between the quarter revivals are observable, where an oscillation with a strength on the order of 0.1 compared to the isotropic value is present. A fit with the expansion in Eq. (11) is performed, and higher order terms are added. Smaller features in the delay dependence can only be fitted through the addition of higher order terms with $n=2$, 3. Especially, 1/8 revivals at 3 and 5 ps are only fitted with higher order terms. The phase of harmonic 19 as a function of delay is extracted using fast Fourier transformations of the collected Young's double slit fringe pattern. In Fig. 7, the fringe pattern of harmonic 19 is given as a function of delay between the pump and probe. The fringes change position most visibly at the times of $4.1 \mathrm{ps}$ and $12.2 \mathrm{ps}$ in the given pattern. With a fringe spacing of 8 pixels, a change in phase of $\pi / 4=0.79 \mathrm{rad}$ for this harmonics is given for each pixel the fringe pattern moves. In the experiment, the observed phase change is on the order of $0.5 \mathrm{rad}$ in the fast Fourier transformation and the equivalent pixel shift is on the order of $2 / 3$ pixel for the shown harmonic, which we can resolve based on the fact that multiple oscillations occur and allow us to have higher resolution sampling of the fringe's movement. Estimates for the resolution can be made in accordance with Ref. 48 . We perform a series of Fourier transformations for all delays and harmonic orders and extract the phase of individual harmonics as a function

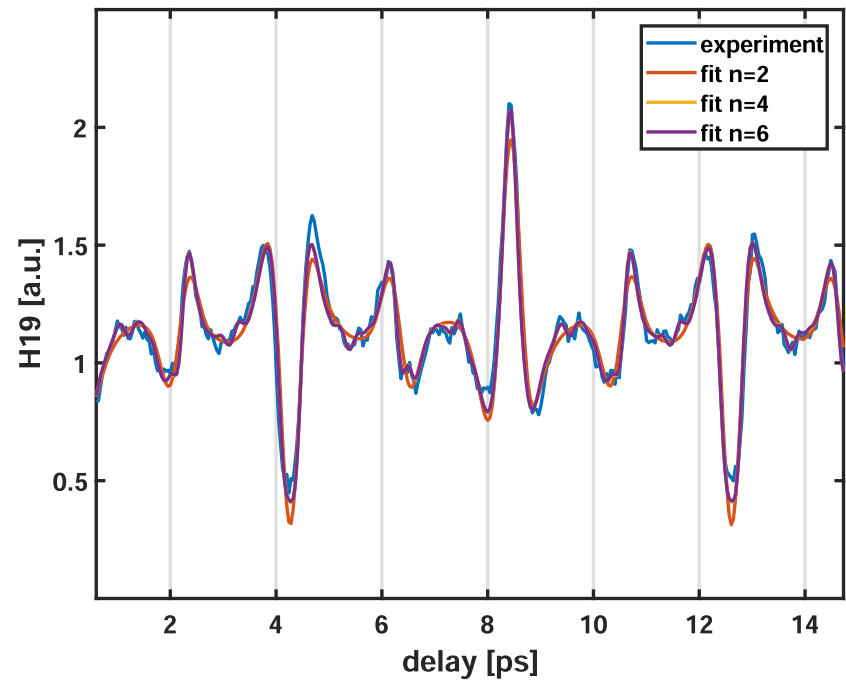

FIG. 6. Amplitude of harmonic 19 recorded as a function of time between pump and probe pulses. The experimental data are fitted to an expansion of varying order.

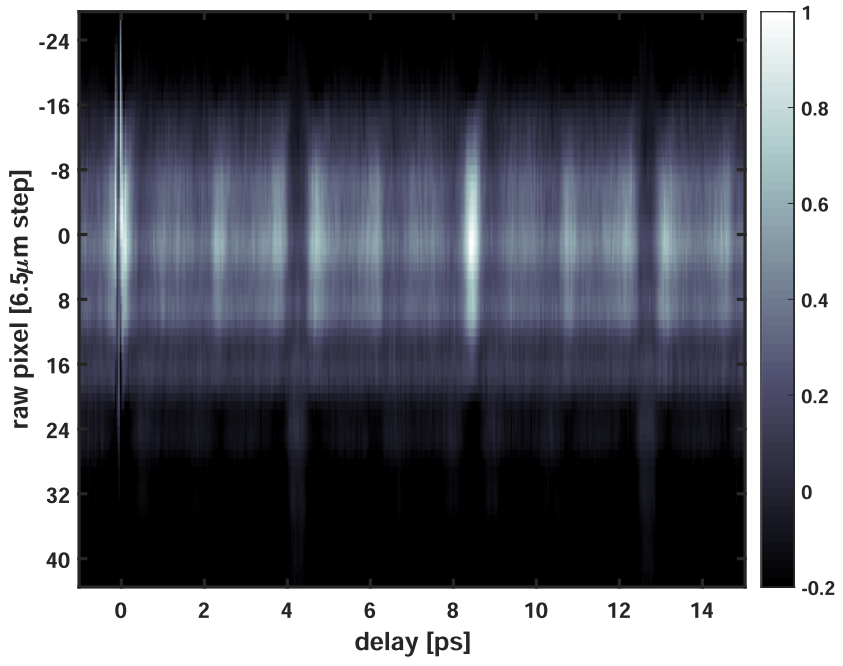

FIG. 7. Interference pattern of harmonic 19 generated by two intense laser foci. The projection is recorded as a function of time between the pump and probe. The fringe spacing is 8 pixels, which equals to $2 \pi$ in phase. A shift of 1 pixel equals a phase change of $0.78 \mathrm{rad}$.

of time. From the measured interference, we obtain a complex valued quantity with $S(\omega, t)=\sqrt{P(\omega, t)} e^{i \phi(\omega, t)}$, in which the imaginary and complex part depend on the phase and amplitude measured in the experiment. To perform a linear regression, the complex number is split into the real and imaginary parts and two linear regressions are performed as the equation splits into two linear equations. After the linear regressions, we convert the complex numbers back into amplitude and phase. In Fig. 8, we show the time-dependent, measured phases of harmonic 9,13,17, and 19. At times of alignment and antialignment, the biggest phase offset compared to the

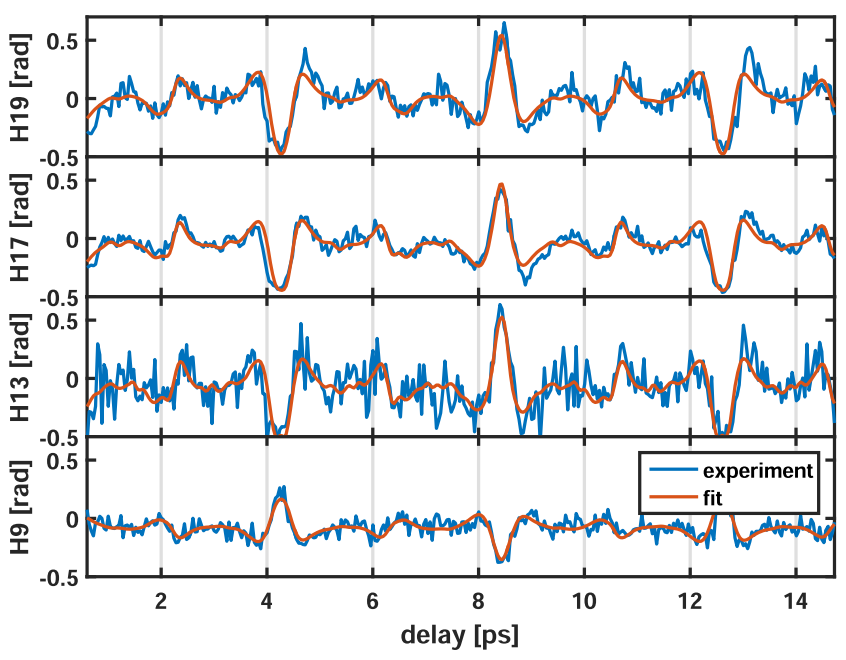

FIG. 8. Phase of harmonic 9,15 , and 19 as a function of time between pump and probe pulses. A fit, based on estimates for the alignment distribution, is shown. 
reference source is observed. The measured phase of harmonic 9 shows a maximum in phase at a delay of $4.1 \mathrm{ps}$, while higher order harmonics show a minimum in phase at this delay, as previously reported by Refs. 25 and 49. No higher order features, e.g., 1/8 revivals, are present in the time dependent phase of the recorded harmonics but changes in phase can be measured at the times of quarter and half-revivals. Harmonic 19 shows a variation of up to 1 $\mathrm{rad}$ as a function of time to the isotropic value. The shown fits, based on a least square method, show agreement with the measurement, however, do not match the width of the peaks at times of antialignment at 4.1 ps and 12.4 ps. The experimental curves show a broader feature in time than the fits can re-produce.

\section{ANGULAR CONTRIBUTIONS}

The angle dependent amplitude and phase in the molecular Eq. (6) frame is plotted in Fig. 9. To calculate the quantity $S(\theta, \omega)$, we used the extracted coefficients $C_{n}$, as detailed in Eq. (11). As mentioned before, both $S(\theta, \omega)$ and $C_{n}$ are complex valued. We observe in the left panel an angle dependent amplitude that has a maximum at $0^{\circ}$ and a local minimum at $90^{\circ}$. As the harmonic order increases, so does the maximum harmonic amplitude. For the 9th and 11th order, we observe a maximum of 2 , while for $\mathrm{H} 17$, we observe a maximum of 4 and for $\mathrm{H} 19$ a maximum of 6 , normalized to its original isotropic value. The extracted phase of the recorded harmonics shows a similar behavior. As the harmonic order increases, so does the absolute change in phase between $0^{\circ}$ and $90^{\circ}$ for the different orders. In the angle-dependent phase, we can observe another feature. At $90^{\circ}$, a local maximum in the extracted phase values is visible. Harmonic 9 shows the opposite angle-dependent behavior as mentioned earlier in the delay dependent phase measurements. Harmonic order 19 is showing a strong angle dependence in amplitude with an angle-resolved amplitude of six times the isotropic value at an angle of $\theta=0^{\circ}$. A phase difference of $1.6 \mathrm{rad}$ between harmonic emission at $\theta=0^{\circ}$ and $\theta=90^{\circ}$ is visible. This feature can be explained with the shape resonance in the photoionization cross section of the HOMO at the particular photon energy. The residuals for the imaginary and real part of the time dependent signal is reducing with order, when higher order terms are being added. The addition of terms of order $2 n=6$ do not improve the fit to the experimental data. Orders $2 n=4$ and $2 n=6$ can predict smaller fractional revivals and do not differ drastically for the given temperature and pulse intensities so that a fit to $2 n=6$ does not improve the delay dependent fit to the experimental data. In Fig. 10, the extracted phase and amplitude of harmonic 17 is given as a function of angle between the molecular axis and the driving laser polarization. We compare the angle-dependence to the angle dependence calculated by the factorization in QRS,

$$
\begin{aligned}
D_{\text {total }}(\omega, \theta)= & \left(N_{\text {Номо }}(\theta)\right)^{1 / 2} d_{\text {номо }}(\omega, \theta) \\
& +\left(N_{\text {HОМО- } 1}(\theta)\right)^{1 / 2} d_{\text {HОМО- }}(\omega, \theta) e^{i \Delta \eta},
\end{aligned}
$$

where $D_{\text {total }}(\omega, \theta)$ is the coherent sum of harmonic dipoles from HOMO and HOMO-1 with the ionization potential difference of HOMO and HOMO-1 of $1.3 \mathrm{eV}$. The ionization rates $N(\theta)$ are given by a theoretical ionization calculation of HOMO and HOMO- 1 by MO-ADK theory extracted from Ref. 50 and $d(\omega, \theta)$ is supplied from Ref. 20 with $d=\sqrt{\sigma} e^{i \phi}$. For the ionization rates
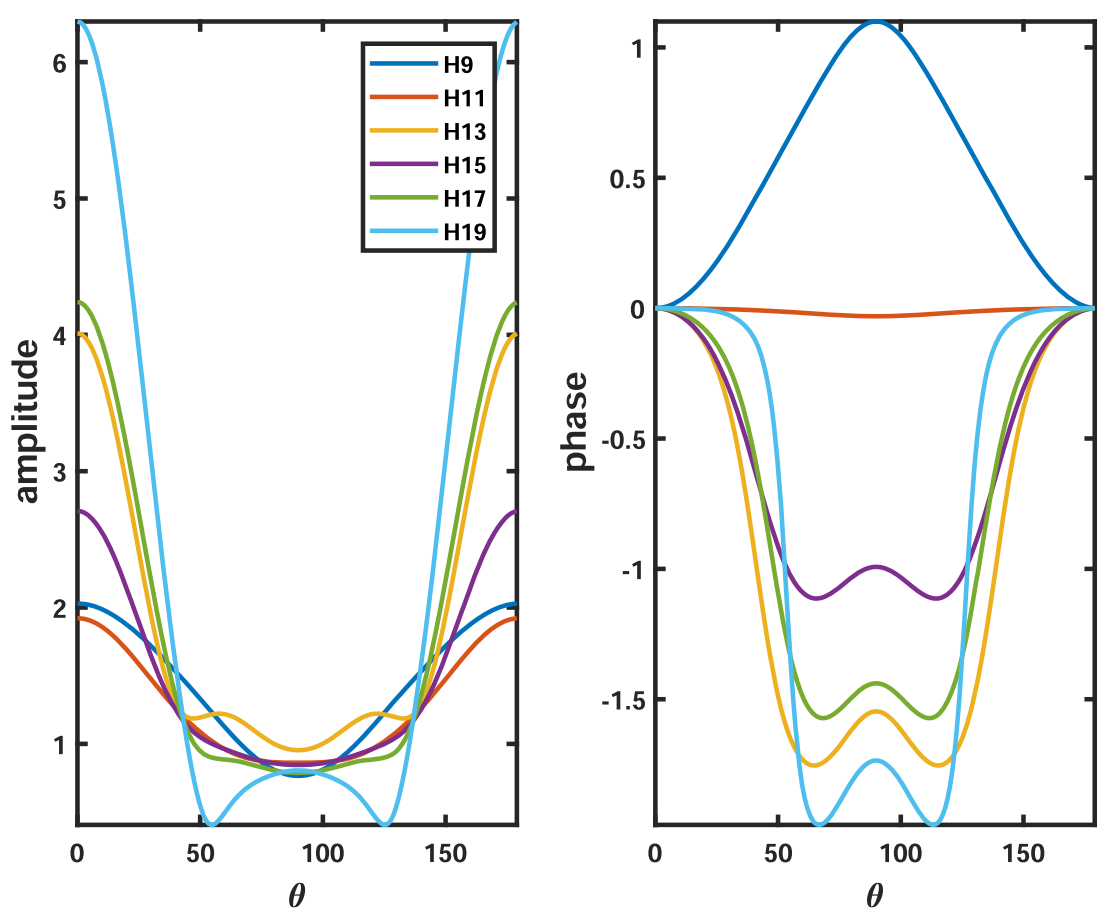

FIG. 9. Phase and amplitude of the harmonic emission in the molecular frame as a function of angle between probe polarization and molecular axis for measured harmonics. 

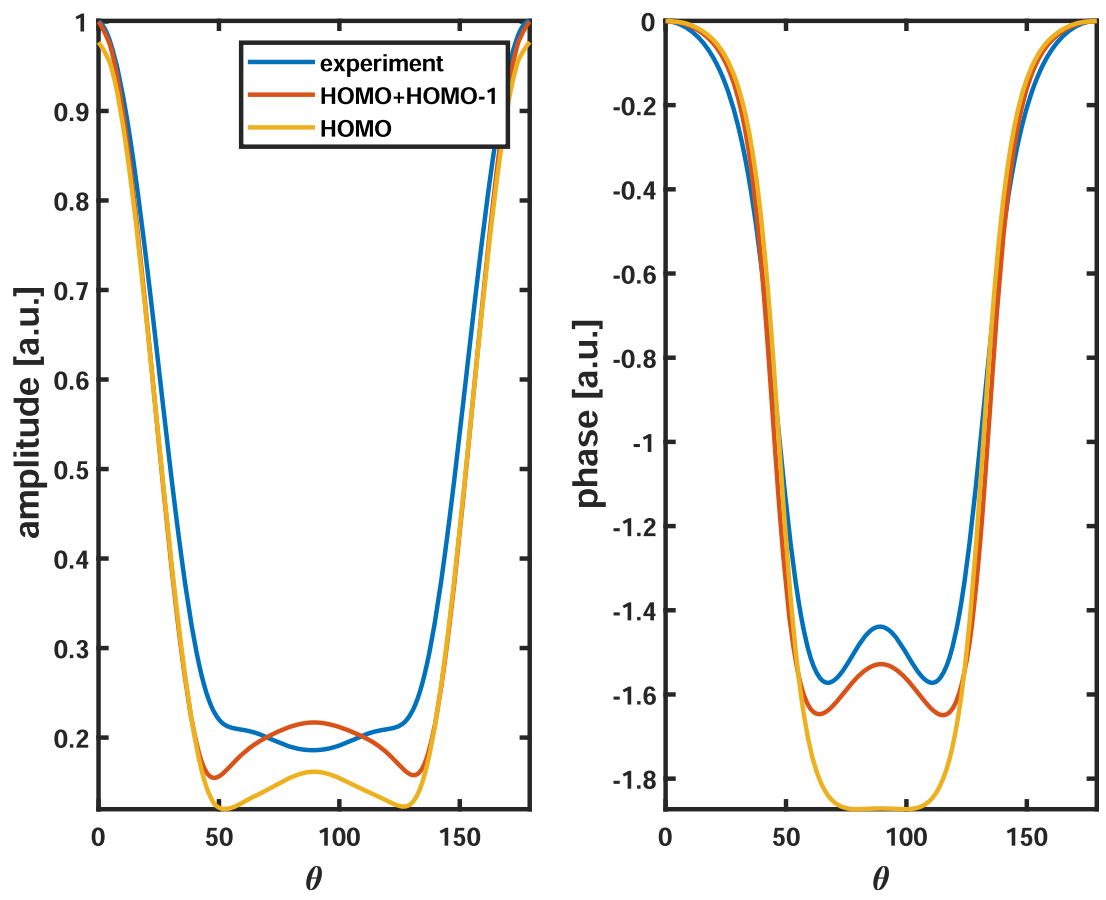

FIG. 10. Harmonic amplitude and phase of $\mathrm{H} 17$ as a function of angle $\theta$. The experimentally retrieved angle dependent phase and amplitude is compared to harmonic emission calculated by the factorization of the harmonic yield in ionization rate, given by MO-ADK, ${ }^{50}$ the phase difference due to the difference in ionization potential and the complex photoionization cross section.

of HOMO and HOMO-1, we use a ratio of 5:1 for the preferential ionization of HOMO over HOMO- 1 at $90^{\circ}$. The angle dependent ionization rate for HOMO has a ratio of 9:1 for ionizing parallel to the molecular axis compared to ionizing perpendicular to the molecular axis. A phase difference given by the classical action of the electron in the continuum is given by the ionization potential difference between the two molecular orbitals and is accounted for by $e^{i \Delta \eta}$. The theoretical photoionization cross section (PICS) calculation then allows us to calculate the harmonic dipole as a product of the given complex-valued amplitudes of ionization rate, PICS, and electron wave packet. The harmonic dipoles are plotted for harmonic order 17 in Fig. 10. The persistent feature visible in the angle dependent phase measurement at $90^{\circ} \mathrm{can}$ be explained only by using nonvanishing probabilities of HOMO-1 to the total harmonic dipole. We can match the retrieved angle dependent phase of harmonic 15 and 17. Harmonic 19 can be explained by HOMO only but shows better agreement with the experiment, when a portion of HOMO-1 is added to the calculated total dipole. Here, we assumed an ionization rate similar to the MO-ADK model rate given by publication, ${ }^{50}$ where the ratio of parallel to the perpendicular ionization rate of HOMO is given with 10:1. However, rates of $3.3: 1^{51}$ and $4.5: 1^{52}$ have been measured. Since we did not measure the angle-dependent single-ionization yield in our experiment, we used the MO-ADK. ${ }^{54-80}$

\section{CONCLUDING REMARKS}

Using OAM to generate two tightly spaced foci has proven a reliable and stable interferometric setup. Since the two beams with opposite OAM are sampled over the whole SLM surface, the interferometer is very robust to external perturbations. Furthermore, thanks to extra degrees of control introduced by the SLM aligning the experiment is very trivial compared with other multibeam setups. Using a combination of OAM and a flat phase $l=1,0$ allows us to experimentally verify the proper overlap between the two sources and the aligning pump pulses. Therefore, these new sets of multibeam experiments, controlled by a SLM, provide a very viable platform for homodyne measurements where the local oscillator is the XUV beam where no pump is present.

Our experimental findings fit well with previous experimental findings. As we did before in a phase matching dependence study, ${ }^{28}$ we observe a strong signature from the lower lying orbital HOMO-1. This time, this feature is very significant in the retrieved angular distributions. This new sensitivity is due in part to the fact that we are using a homodyne measurement. To the best of our knowledge, this is the first time that a measurement has been performed to retrieve the photoionization phase of the $\mathrm{N}_{2} \mathrm{HOMO}-1$ orbital.

In this experiment, we report HHG from HOMO and HOMO-1 for low order harmonics, which match the characteristic features of the photoionization cross section in phase and amplitude. In previous work, ${ }^{53}$ features from HOMO-1 in nitrogen were restricted to cut-off harmonics with photon energies of harmonic order 35 and higher. Here, we report low order harmonics in nitrogen that are generated from HOMO- 1 .

\section{ACKNOWLEDGMENTS}

We would like to thank V. Kumarappan, X. Ren, V. Makhija, and A. Thu-Le for useful discussions. This work was supported by the Chemical Sciences, Geosciences, and Biosciences Division, Office of Basic Energy Sciences, Office of Science, U.S. Department of Energy (DOE) under Grant No. DE-FG02-86ER13491. 
C.A.T.-H. was partially funded by the Chemical Sciences, Geosciences, and Biosciences Division, Office of Basic Energy Sciences, Office of Science, U.S. Department of Energy (DOE) Grant No. DE-SC0019098.

\section{REFERENCES}

${ }^{1}$ P. B. Corkum, Phys. Rev. Lett. 71, 1994 (1993).

${ }^{2}$ J. L. Krause, K. J. Schafer, and K. C. Kulander, Phys. Rev. Lett. 68, 3535 (1992).

${ }^{3}$ A. L'Huillier and P. Balcou, Phys. Rev. Lett. 70, 774 (1993).

${ }^{4}$ A. McPherson, G. Gibson, H. Jara, U. Johann, T. S. Luk, I. McIntyre, K. Boyer, and C. K. Rhodes, J. Opt. Soc. Am. B 4, 595 (1987).

${ }^{5}$ M. Ferray, A. L'Huillier, X. Li, L. Lompre, G. Mainfray, and C. Manus, J. Phys. B: At., Mol. Opt. Phys. 21, L31 (1988).

${ }^{6}$ S. Baker, J. S. Robinson, C. Haworth, H. Teng, R. Smith, C. Chirilă, M. Lein, J. Tisch, and J. P. Marangos, Science 312, 424 (2006).

${ }^{7}$ J. Itatani, J. Levesque, D. Zeidler, H. Niikura, H. Pépin, J.-C. Kieffer, P. B. Corkum, and D. M. Villeneuve, Nature 432, 867 (2004).

${ }^{8}$ M. Lein, N. Hay, R. Velotta, J. P. Marangos, and P. L. Knight, Phys. Rev. A 66, 023805 (2002).

${ }^{9}$ R. Velotta, N. Hay, M. B. Mason, M. Castillejo, and J. P. Marangos, Phys. Rev. Lett. 87, 183901 (2001).

${ }^{10}$ T. Kanai, S. Minemoto, and H. Sakai, Nature 435, 470 (2005).

${ }^{11}$ X. Zhou, R. Lock, W. Li, N. Wagner, M. M. Murnane, and H. C. Kapteyn, Phys. Rev. Lett. 100, 073902 (2008).

${ }^{12}$ Y. Mairesse and F. Quéré, Phys. Rev. A 71, 011401 (2005).

${ }^{13}$ O. Smirnova, Y. Mairesse, S. Patchkovskii, N. Dudovich, D. Villeneuve, P. Corkum, and M. Y. Ivanov, Nature 460, 972 (2009).

${ }^{14}$ H. J. Wörner, J. B. Bertrand, P. Hockett, P. B. Corkum, and D. M. Villeneuve, Phys. Rev. Lett. 104, 233904 (2010).

${ }^{15}$ P. M. Kraus, B. Mignolet, D. Baykusheva, A. Rupenyan, L. Hornỳ, E. F. Penka, G. Grassi, O. I. Tolstikhin, J. Schneider, F. Jensen et al., Science 350, 790 (2015).

${ }^{16}$ A. D. Shiner, B. E. Schmidt, C. Trallero-Herrero, H. J. Wörner, S. Patchkovskii, P. B. Corkum, J.-C. Kieffer, F. Légaré, and D. M. Villeneuve, Nat. Phys. 7, 464 (2011).

${ }^{17}$ L. D. Landau and E. M. Lifshitz, Quantum Mechanics, Nonrelativistic Theory (Pergamon, Oxford, 1977).

${ }^{18}$ M. V. Frolov, N. L. Manakov, T. S. Sarantseva, M. Y. Emelin, M. Y. Ryabikin, and A. F. Starace, Phys. Rev. Lett. 102, 243901 (2009).

${ }^{19}$ M. Frolov, T. Sarantseva, N. Manakov, K. Fulfer, B. Wilson, J. Troß, X. Ren, E. D. Poliakoff, A. Silaev, N. Vvedenskii et al., Phys. Rev. A 93, 031403 (2016).

${ }^{20} \mathrm{C}$. Jin, J. B. Bertrand, R. Lucchese, H. Wörner, P. B. Corkum, D. Villeneuve, A.-T. Le, and C. D. Lin, Phys. Rev. A 85, 013405 (2012).

${ }^{21}$ P. M. Paul, E. Toma, P. Breger, G. Mullot, F. Augé, P. Balcou, H. Muller, and P. Agostini, Science 292, 1689 (2001).

${ }^{22}$ H. Muller, Appl. Phys. B 74, s17 (2002).

${ }^{23}$ A. Ferré, A. E. Boguslavskiy, M. Dagan, V. Blanchet, B. D. Bruner, F. Burgy, A. Camper, D. Descamps, B. Fabre, N. Fedorov, J. Gaudin, G. Geoffroy, J. Mikosch, S. Patchkovskii, S. Petit, T. Ruchon, H. Soifer, D. Staedter, I. Wilkinson, A. Stolow, N. Dudovich, and Y. Mairesse, Nat. Commun. 6, 5952 (2015).

${ }^{24}$ R. M. Lock, X. Zhou, W. Li, M. M. Murnane, and H. C. Kapteyn, Chem. Phys. 366, 22 (2009).

${ }^{25}$ A. Camper, A. Ferré, N. Lin, E. Skantzakis, D. Staedter, E. English, B. Manschwetus, F. Burgy, S. Petit, D. Descamps et al., Photonics (Multidisciplinary Digital Publishing Institute, 2015), Vol. 2, pp. 184-199.

${ }^{26}$ B. K. McFarland, J. P. Farrell, P. H. Bucksbaum, and M. Gühr, Science 322, 1232 (2008).

${ }^{27}$ A. Rupenyan, J. B. Bertrand, D. M. Villeneuve, and H. J. Wörner, Phys. Rev. Lett. 108, 033903 (2012).

${ }^{28}$ J. Troß, X. Ren, V. Makhija, S. Mondal, V. Kumarappan, and C. A. TralleroHerrero, Phys. Rev. A 95, 033419 (2017).

${ }^{29}$ K. Fukui, T. Yonezawa, and H. Shingu, J. Chem. Phys. 20, 722 (1952).
${ }^{30}$ J. C. Slater, Phys. Rev. 81, 385 (1951).

${ }^{31}$ C. Z. Bisgaard, M. D. Poulsen, E. Péronne, S. S. Viftrup, and H. Stapelfeldt, Phys. Rev. Lett. 92, 173004 (2004).

${ }^{32}$ J. P. Cryan, P. H. Bucksbaum, and R. N. Coffee, Phys. Rev. A 80, 063412 (2009).

${ }^{33}$ X. Ren, V. Makhija, A.-T. Le, J. Troß, S. Mondal, C. Jin, V. Kumarappan, and C. Trallero-Herrero, Phys. Rev. A 88, 043421 (2013).

${ }^{34}$ P. M. Kraus, D. Baykusheva, and H. J. Wörner, Phys. Rev. Lett. 113, 023001 (2014).

${ }^{35}$ X. Ren, V. Makhija, H. Li, M. F. Kling, and V. Kumarappan, Phys. Rev. A 90, 013419 (2014).

${ }^{36}$ D. Irimia, D. Dobrikov, R. Kortekaas, H. Voet, D. A. van den Ende, W. A. Groen, and M. H. Janssen, Rev. Sci. Instrum. 80, 113303 (2009).

${ }^{37}$ D. G. Voelz, Computational Fourier Optics: A MATLAB Tutorial (SPIE Press Bellingham, Washington, USA, 2011).

${ }^{38}$ G. Gariepy, J. Leach, K. T. Kim, T. Hammond, E. Frumker, R. W. Boyd, and P. Corkum, Phys. Rev. Lett. 113, 153901 (2014).

${ }^{39}$ X. Yu, C. A. Trallero-Herrero, and S. Lei, Appl. Surf. Sci. 360, 833 (2016).

${ }^{40}$ L. Allen, M. W. Beijersbergen, R. Spreeuw, and J. Woerdman, Phys. Rev. A 45, 8185 (1992).

${ }^{41}$ R. Bourouis, K. A. Ameur, and H. Ladjouze, J. Mod. Opt. 44, 1417 (1997)

${ }^{42}$ N. Passilly, F. Treussart, R. Hierle, R. de Saint Denis, K. Ait-Ameur, and J.-F. Roch, J. Opt. Soc. Am. A 22, 984 (2005).

${ }^{43}$ N. Smith, W. Heathcote, G. Hechenblaikner, E. Nugent, and C. Foot, J. Phys. B: At., Mol. Opt. Phys. 38, 223 (2005).

${ }^{44}$ T. Meyrath, F. Schreck, J. Hanssen, C.-S. Chuu, and M. Raizen, Opt. Express 13, 2843 (2005).

${ }^{45}$ J. Tross, G. Kolliopoulos, and C. A. Trallero-Herrero, Opt. Express 27, 22960 (2019).

${ }^{46}$ A.-T. Le, R. Lucchese, S. Tonzani, T. Morishita, and C. Lin, Phys. Rev. A 80, 013401 (2009).

${ }^{47}$ S. Ramakrishna and T. Seideman, Phys. Rev. Lett. 99, 113901 (2007).

${ }^{48}$ D. J. Bone, H.-A. Bachor, and R. J. Sandeman, Appl. Opt. 25, 1653 (1986).

${ }^{49}$ H. Soifer, P. Botheron, D. Shafir, A. Diner, O. Raz, B. Bruner, Y. Mairesse, B. Pons, and N. Dudovich, Phys. Rev. Lett. 105, 143904 (2010).

${ }^{50}$ A.-T. Le, R. Lucchese, and C. Lin, J. Phys. B: At., Mol. Opt. Phys. 42, 211001 (2009).

${ }^{51}$ D. Pavičić, K. F. Lee, D. M. Rayner, P. B. Corkum, and D. M. Villeneuve, Phys. Rev. Lett. 98, 243001 (2007).

${ }^{52}$ I. V. Litvinyuk, K. F. Lee, P. W. Dooley, D. M. Rayner, D. M. Villeneuve, and P. B. Corkum, Phys. Rev. Lett. 90, 233003 (2003).

${ }^{53}$ B. K. McFarland, J. Farrell, P. Bucksbaum, and M. Gühr, Phys. Rev. A 80, 033412 (2009).

${ }^{54}$ M. Schultze, M. Fieß, N. Karpowicz, J. Gagnon, M. Korbman, M. Hofstetter, S. Neppl, A. L. Cavalieri, Y. Komninos, T. Mercouris et al., Science 328, 1658 (2010).

${ }^{55}$ M. Lein, N. Hay, R. Velotta, J. P. Marangos, and P. L. Knight, Phys. Rev. Lett. 88, 183903 (2002).

${ }^{56}$ T. Morishita, A.-T. Le, Z. Chen, and C. D. Lin, Phys. Rev. Lett. 100, 013903 (2008).

${ }^{57}$ X. Ren, V. Makhija, and V. Kumarappan, Phys. Rev. A 85, 033405 (2012).

${ }^{58}$ J. B. Bertrand, H. J. Wörner, P. Hockett, D. M. Villeneuve, and P. B. Corkum, Phys. Rev. Lett. 109, 143001 (2012).

${ }^{59}$ R. Zerne, C. Altucci, M. Bellini, M. B. Gaarde, T. Hänsch, A. L’Huillier, C. Lyngå, and C.-G. Wahlström, Phys. Rev. Lett. 79, 1006 (1997).

${ }^{60}$ M. Lewenstein, P. Balcou, M. Y. Ivanov, A. L'huillier, and P. B. Corkum, Phys. Rev. A 49, 2117 (1994).

${ }^{61}$ P. Antoine, A. L'huillier, and M. Lewenstein, Phys. Rev. Lett. 77, 1234 (1996).

${ }^{62}$ Y. Nabekawa, T. Shimizu, T. Okino, K. Furusawa, H. Hasegawa, K. Yamanouchi, and K. Midorikawa, Phys. Rev. Lett. 97, 153904 (2006).

${ }^{63}$ Y. Nabekawa, H. Hasegawa, E. J. Takahashi, and K. Midorikawa, Phys. Rev. Lett. 94, 043001 (2005). 
${ }^{64}$ J. Mauritsson, P. Johnsson, E. Gustafsson, A. L'Huillier, K. Schafer, and M. Gaarde, Phys. Rev. Lett. 97, 013001 (2006).

${ }^{65}$ E. Constant, V. D. Taranukhin, A. Stolow, and P. Corkum, Phys. Rev. A 56, 3870 (1997).

${ }^{66}$ G. Sansone, E. Benedetti, F. Calegari, C. Vozzi, L. Avaldi, R. Flammini, L. Poletto, P. Villoresi, C. Altucci, R. Velotta et al., Science 314, 443 (2006).

${ }^{67}$ M. Hentschel, R. Kienberger, C. Spielmann, G. A. Reider, N. Milosevic, T. Brabec, P. Corkum, U. Heinzmann, M. Drescher, and F. Krausz, Nature 414, 509 (2001).

${ }^{68} \mathrm{~K}$. Klünder, J. Dahlström, M. Gisselbrecht, T. Fordell, M. Swoboda, D. Guenot, P. Johnsson, J. Caillat, J. Mauritsson, A. Maquet et al., Phys. Rev. Lett. 106, 143002 (2011).

${ }^{69}$ P. B. Corkum and F. Krausz, Nat. Phys. 3, 381 (2007).

${ }^{70}$ P. Tzallas, D. Charalambidis, N. Papadogiannis, K. Witte, and G. D. Tsakiris, Nature 426, 267 (2003).

${ }^{71}$ J. Itatani, F. Quéré, G. L. Yudin, M. Y. Ivanov, F. Krausz, and P. B. Corkum, Phys. Rev. Lett. 88, 173903 (2002).

${ }^{72}$ R. Kienberger, E. Goulielmakis, M. Uiberacker, A. Baltuska, V. Yakovlev, F. Bammer, A. Scrinzi, T. Westerwalbesloh, U. Kleineberg, U. Heinzmann et al., Nature 427, 817 (2004).
${ }^{73}$ A. Baltuška, T. Udem, M. Uiberacker, M. Hentschel, E. Goulielmakis, C. Gohle, R. Holzwarth, V. Yakovlev, A. Scrinzi, T. W. Hänsch et al., Nature 421, 611 (2003).

${ }^{74}$ M. Ossiander, F. Siegrist, V. Shirvanyan, R. Pazourek, A. Sommer, T. Latka, A. Guggenmos, S. Nagele, J. Feist, J. Burgdörfer et al., Nat. Phys. 13, 280 (2016).

${ }^{75}$ H. Bandulet, D. Comtois, A. Shiner, C. Trallero-Herrero, N. Kajumba, T. Ozaki, P. Corkum, D. Villeneuve, J. Kieffer, and F. Légaré, J. Phys. B: At., Mol. Opt. Phys, 41, 245602 (2008).

${ }^{76}$ J. Bertrand, H. Wörner, P. Salières, D. Villeneuve, and P. Corkum, Nat. Phys. 9 , 174 (2013).

${ }^{77}$ M. Bellini, C. Lyngå, A. Tozzi, M. Gaarde, T. Hänsch, A. L'Huillier, and C.-G. Wahlström, Phys. Rev. Lett. 81, 297 (1998).

${ }^{78}$ A. Rundquist, C. G. Durfee, Z. Chang, C. Herne, S. Backus, M. M. Murnane, and H. C. Kapteyn, Science 280, 1412 (1998).

${ }^{79}$ M. Nisoli, E. Priori, G. Sansone, S. Stagira, G. Cerullo, S. De Silvestri, C. Altucci, R. Bruzzese, C. De Lisio, P. Villoresi et al., Phys. Rev. Lett. 88, 033902 (2002).

${ }^{80}$ B. Langdon, J. Garlick, X. Ren, D. J. Wilson, A. M. Summers, S. Zigo, M. F. Kling, S. Lei, C. G. Elles, E. Wells et al., Opt. Express 23, 4563 (2015). 